\title{
How Do Electric and Magnetic Fields Move?
}

\author{
Vladimir Alexander Leus \\ Department of Electrical Engineering and Electronics, University of Liverpool, Liverpool, UK \\ Email: vladalex@liv.ac.uk
}

How to cite this paper: Leus, V.A. (2020) How Do Electric and Magnetic Fields Move? Journal of Modern Physics, 11, 281-284.

https://doi.org/10.4236/jmp.2020.112016

Received: January 21, 2020

Accepted: February 17, 2020

Published: February 20, 2020

Copyright $\odot 2020$ by author(s) and Scientific Research Publishing Inc. This work is licensed under the Creative Commons Attribution International License (CC BY 4.0).

http://creativecommons.org/licenses/by/4.0/

\begin{abstract}
In nature, there are two fundamentally different types of motion of the electric and magnetic fields: dynamic and kinematic. A typical manifestation of the first type of motion takes place in a plane harmonic EM-wave. For already more than a century the question about the ratio of the phases of the electric and magnetic fields, oscillating in such a wave, remains open. From time to time in this regard, fierce disputes arise. The point is that far from any phase difference turns out to be compatible with the full system of Maxwellian equations. Maxwell's classical theory as applied to such a wave leads to the conclusion that the electric and magnetic vectors in it oscillate harmoniously with zero phase shift. In the framework of this theory, a rigorous mathematical proof is given.
\end{abstract}

\section{Keywords}

Electromagnetic Wave, Transverse Oscillations in Phase, Longitudinal Immobility of Field Vectors, Electro-Kinematics, Magneto-Kinematics

\section{Introduction}

After Heinrich Hertz, a famous German physicist, discovered experimentally the existence of electromagnetic waves propagating in vacuum, all physicists believed that the movement of energy in space occurs due to the periodic process of mutual induction of electric and magnetic fields. The following picture of the phenomenon looked quite logical. When the magnetic field at a given point in space decreases, the electric field increases, reaching a maximum on the moment of zeroing the magnetic, and vice versa. The zero value of the magnetic vector corresponds to the maximum rate of its change, which in turn implies the maximum value of the induced electric vector, and also vice versa. The beautiful idea of a mutual transfer of energy from a magnetic form to an electric one and back proved to be extremely tenacious. Outbreaks of this "faith" sometimes still occur [1], although rigorous proof of its falsity has already been found. 
Too scholastic, schooling-wise, even trivial as this might seem, the question about phase shift between electric and magnetic vectors in EM-wave has a peculiar, just Shakespearian tension for the Maxwell's theory of electromagnetism: “To be" -Figure 1(a), or "not to be" -Figure 1(b).

\section{Solution of the Problem}

Show that in a plane harmonic EM-wave propagating in a vacuum, there is no periodic conversion of energy between magnetism and electricity. It is known that in such a wave, the electric and magnetic vectors are orthogonal to the direction of propagation and mutually orthogonal, therefore, we choose the coordinate system in the following way. The $x$ axis is oriented along the propagation direction, the $y$ axis is parallel to the electric vector, the $z$ axis is parallel to the magnetic vector so that there is a right-oriented triple of unit basis vectors (Figure $1)$. In this system, the electric vector $E$ has coordinates $(0, E, 0)$, and the magnetic one $\mathrm{H}$ has coordinates $(0,0, H)$. The "electric" and "magnetic" coordinates depend on time and space according to a harmonic law. We can take $E=E_{0} \cos \left(\omega t-\frac{\omega}{c} x\right)$, then $H=H_{0} \cos \left(\omega t-\frac{\omega}{c} x+\delta\right)$. Here, $E_{0}$ and $H_{0}$ are half-amplitude values, and $\delta$ is an unknown phase shift.

Since there are no currents in a vacuum, both vectors obey two Maxwell equations, which in a Gaussian system of units have the form:

$$
\operatorname{curl} \mathbf{E}=-\frac{1}{c} \frac{\partial \mathbf{H}}{\partial t}, \operatorname{curl} \mathbf{H}=\frac{1}{c} \frac{\partial \mathbf{E}}{\partial t} .
$$

In the orthonormal coordinate system $(x, y, z)$, the curl of the vector field $\mathbf{F}=\left(F_{x}, F_{y}, F_{z}\right)$ is a vector whose coordinates are expressed in terms of the spatial partial derivatives of the coordinates of the field vector as follows:

$$
\operatorname{curl} \mathbf{F}=\left(\frac{\partial F_{z}}{\partial y}-\frac{\partial F_{y}}{\partial z}, \frac{\partial F_{x}}{\partial z}-\frac{\partial F_{z}}{\partial x}, \frac{\partial F_{y}}{\partial x}-\frac{\partial F_{x}}{\partial y}\right) .
$$

In our case, from all the spatial partial derivatives of the electric vector, non-zero one would be only

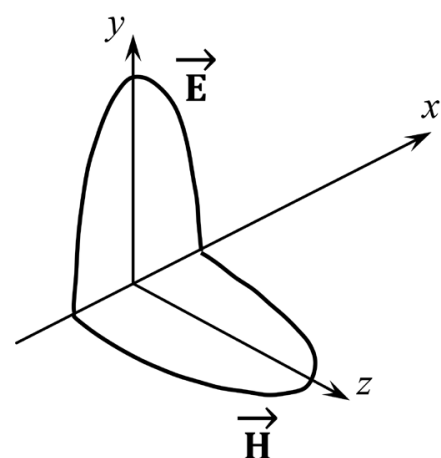

(a)

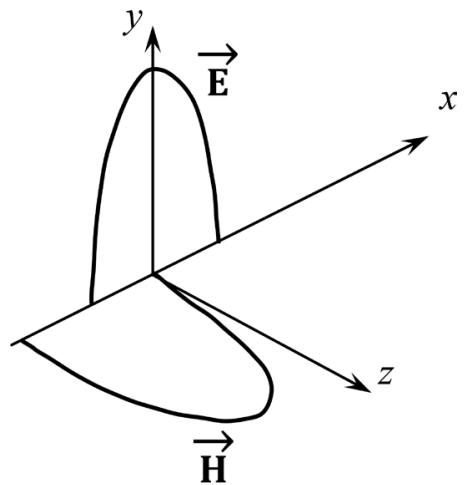

(b)

Figure 1. Phase shift in EM-wave. 


$$
\frac{\partial E_{y}}{\partial x}=\frac{\omega}{c} E_{0} \sin \left(\omega t-\frac{\omega}{c} x\right)
$$

therefore vector

$$
\operatorname{curl} \mathbf{E}=\left(0,0, \frac{\omega}{c} E_{0} \sin \left(\omega t-\frac{\omega}{c} x\right)\right)
$$

Let us find the partial time derivative of the magnetic vector. Since only the third its coordinate is different from zero, then

$$
\frac{\partial \mathbf{H}}{\partial t}=\left(0,0,-\omega H_{0} \sin \left(\omega t-\frac{\omega}{c} x+\delta\right)\right) .
$$

From a comparison of (1) and (2), it is seen that a necessary and sufficient condition for the satisfying of the first of the two above Maxwell equations is a multiple of the constant $2 \pi$ value of the phase shift $\delta=0, \pm 2 \pi, \pm 4 \pi, \cdots$.

Let us verify this condition for the second of the equations. We have

$$
\operatorname{curl} \mathbf{H}=\left(0,-\frac{\partial H_{z}}{\partial x}, 0\right)=\left(0,-\frac{\omega}{c} H_{0} \sin \left(\omega t-\frac{\omega}{c} x+\delta\right), 0\right),
$$

and

$$
\frac{\partial \mathbf{E}}{\partial t}=\left(0,-\omega E_{0} \sin \left(\omega t-\frac{\omega}{c} x\right), 0\right)
$$

Comparing between (3) and (4) shows the fairness of the above mentioned condition also for the second of Maxwell's equations. Thus, we can draw the following conclusion. In a plane harmonic EM wave propagating in vacuum, the electric and magnetic vectors oscillate in phase. At any point in space, they simultaneously pass through zero and simultaneously reach their semi-amplitude values ( $E_{0}=H_{0}$ in the Gaussian system of units). A complete energy exchange between the electric and magnetic components requires a specific phase shift that is equal to the definite value $\pi / 2$, but Nature does not want to provide even a partial energy transfer between the electric and magnetic forms.

\section{Conclusions}

As we can see, the relationship between the phases of the electric and magnetic vectors in a plane EM wave has far from just a simple "educational" value. The correct solution to this issue is rather of more philosophical, even worldview significance. The adequacy of our perception of the surrounding reality depends on the accepted answer.

Certainly, intuition is a wonderful thing, but, alas, it isn 't omnipotent. In addition to the example of intuitionistic failure discussed above, there is another similar case in the doctrine of electromagnetism. In essence, it has folk rather than scientific character, and consists of a magical belief that the fields in the EM wave move at the speed of light. In this misconception, the main role is played by confusion with energy transfer in space. In fact, electrodynamics implements 
a complete analogy with waves on the surface of a pond. Because of the stone falling into the pond, water moves only in the vertical direction, but these coordinated shifting are transporting horizontally (at a speed of meters per second) the energy that destroys the bank. Similarly, in an EM wave the electric field and magnetic field do not move anywhere in the source's own system, and only their coordinated transverse oscillations provide energy transport in the longitudinal direction [2]. Such behavior of field vectors returns to life the idea of worldwide ether.

As for the true spatial displacements of the electric and magnetic fields, they objectively exist, but are not related to electrodynamics. The study of such movements is carried out in the framework of electro-magneto-kinematics-a specific region in the doctrine of electricity and magnetism [3] [4] [5] [6] [7]. Two Russian engineers, N. Zaev and V. Dokuchaev, opened this new page in the electromagnetic doctrine half a century ago due to an experiment with a rotating electromagnet.

\section{Conflicts of Interest}

The author declares no conflicts of interest regarding the publication of this paper.

\section{References}

[1] Michaud, A. (2020) Journal of Modern Physics, 11, 16-80. https://doi.org/10.4236/jmp.2020.111003

[2] Leus, V.A., Smith, R.T. and Maher, S. (2013) Applied Physics Research, 5, 56-68. https://doi.org/10.5539/apr.v5n4p56

[3] Leus, V.A. and Taylor, S. (2012) Experimental Evidence for the Magneto-Kinematic Effect. PIERS Proceedings, Moscow, 19-23 August 2012, 1040-1048. http://piers.org/pierspublications/PIERS2012MoscowProceedings03.pdf

[4] Leus, V. and Taylor, S. (2011) European Journal of Physics, 32, 1179-1192. https://doi.org/10.1088/0143-0807/32/5/006

[5] Taylor, S. and Leus, V. (2012) European Journal of Physics, 33, 837-852. https://doi.org/10.1088/0143-0807/33/4/837

[6] Leus, V.A. (2015) Applied Physics Research, 7, 95-107. https://doi.org/10.5539/apr.v7n6p95

[7] Leus, V.A. and Taylor, S. (2018) International Journal of Applied Mathematics and Physics, 4, 91-97.

\section{The List of Used Variables}

E -electric vector, $\mathbf{H}$-magnetic vector, $c$-light speed in vacuum, $\omega$ - circular frequency. 